\title{
$616-109$ \\ contribución al estudio de los problemas de analogías creados por los molinos de bolas
}

Michel PAPADAKIS, ingeniero

Publicación Técnica núm. 141 del Centre d’Etudes et de Recherches de l'Industrie des Liants Hydrauliques

\section{Introducción}

Los estudios de molienda efectuados en el C.E.R.I.L.H. desde hace algunos años han permitido poner en evidencia ciertas caracierísticas cuya importancia es notable. Se ha podido comprobar que el modo de trabajo de un molino de bolas, aparentemente complejo, hace intervenir dos propiedades esenciales del material que se muele:

- aptitud a la rotura;

- aptitud a la aglomeración.

La primera propiedad es evidente, pero la segunda lo es menos. Numerosas experiencias confirman siempre su existencia.

El hecho de haber podido esclarecer el comportamiento del material ha facilitado grandemente la comprensićn del funcionamiento de los molinos de bolas. Entre nuestras conclusiones. ya expuestas en el curso de publicaciones anteriores, la más importante es la siguiente:

«Un molino de bolas que trata un cierto material a una finura dada, no puede producir el máximo rendimiento más que para una sola distribución del tamaño de los cuerpos molturadores que encierra.»

Esta conclusión, válida para un material duro tal como el clínker, permite pensar que el molino de bolas en circuito abierto no puede mejorarse indefinidamente y que se encuentra próximo al límite de sus posibilidades.

Se fuede, en principio, prever el comportamiento de tal molino con ayuda de un cierto número de hipótesis y cálculos, de lo que el presente trabajo proporciona una versión mejorada. Este trabajo responde, pues, en primer lugar, a las preocupaciones del usuario, el cual, teniendo un determinado material, trata de utilizarlo lo mejor posible. También esta misma preocupación es la del constructor que trata de dimensionar las máquinas lo más aquilatadas posible por razones económicas evidentes.

La introducción del diámetro del molino como variable importante constituye precisamente el medio de responder a esta cuestión. El problema es siempre complejo y la multiplicidad de las otras variables hacen los cálculos difíciles $\mathrm{y}$, a veces, imposibles. El presente trabajo no pretende resolver este problema. Es preciso considerarlo como una introducción al estudio de la similitud en el dominio de los molinos de bolas.

\section{Notaciones}

Es necesario colocar a la cabeza de la exposición la lista de las notaciones empleadas que se distribuirán en dos categorías: 


\section{1) Notaciones cuya significación es inmediata.}

$\varnothing=$ diámetro del molino (medido sobre revestimiento interior);

$\mathrm{D}=$ diámetro de las bolas;

$\mathrm{N}=$ velocidad de rotación del molino $\left(N=\frac{5,3}{\sqrt{\varnothing}}, \mathrm{N} \mathrm{rev} / \mathrm{s}\right.$ y $\left.\varnothing \mathrm{cm}\right)$

$\mathrm{g}=$ aceleración de la gravedad;

$\mathrm{S}_{p}=$ superficie específica;

$\mathrm{S}_{p i}=$ superficie específica inicial;

$\rho=$ masa volúmica de las bolas;

$\alpha=\frac{\text { peso del material }}{\text { peso de las bolas }}$ (el material deberellenar los huecos existentes entre las bolas, supuestas en reposo).

\section{2) Notaciones a explicar.}

$B=$ coeficiente de aptitud a la rotura.

Se determina efectuando una serie de compresiones seguidas de desaglomeraciones sobre la misma muestra del material. Se utiliza una prensa con ayuda de la cual se establece el diagrama esfuerzo/desplazamiento, tal como se representa en la figura 1. Este diagrama permite medir el trabajo gastado. La nueva superficie específica de la muestra se determina por un método de permeabilidad.

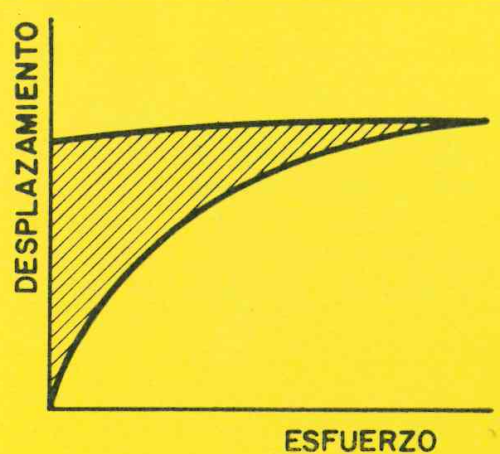

Fig. 1.-Medida del trabajo de compresión.

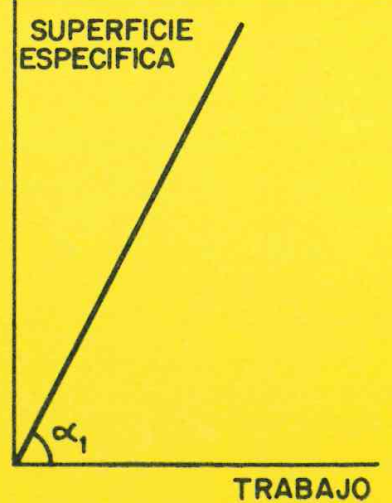

Fig. 2.-Medida del coeficiente B.

Los pares de valores de la superficie específica y del trabajo correspondiente se colocan sobre un gráfico y dan, cuando el material es homogéneo, una relación lineal. Por definición se establece $B=\operatorname{tg} \alpha_{1}$. En C.G.S., $B$ se expresa en $\mathrm{cm}^{2} /$ erg.

$A=$ coeficiente de aptitud a la aglomeración $0<A<1$.

Este coeficiente no se puede medir directamente. Depende simultáneamente de los materiales y del material (ver más adelante).

$A$ es una magnitud sin dimensiones.

$\delta=$ coeficiente de eficacia (sin dimensiones).

Su existencia se justifica por el hecho de que las bolas se entorpecen mutuamente, tanto más cuanto son más gruesas con relación al diámetro del molino. Resulta, pues, que $\delta=f(D / \varnothing)$. $\delta$ tiende hacia 1 cuando $D$ se hace pequeño con relación a $\varnothing$.

$\gamma=$ coeficiente de transmisión de energía (sin dimensiones). 
Este es un moderador de energía cinética cuyo origen no está claro, pero que es indispensable de hacer intervenir en los cálculos para tener cuenta de la experiencia.

$W=$ energía cinética de una bola.

Eligiendo arbitrariamente una altura de caída igual a $0,75 \varnothing$, resulta:

$W=0,39 \rho g \varnothing \gamma D^{3}$ (por aplicación de $W=\frac{1}{2} M V^{2}$ ).

$W_{m}=$ trabajo máximo absorbible durante el aplastamiento de la unidad de masa de los materiales a molturar. $W_{m}$ se deduce del diagrama de la figura 1 (para el clínker se encuentra, por ejemplo, $W_{m}=10^{s} \mathrm{erg} /$ gramo).

\section{Nociones fundamentales}

El molino de bolas trabaja normalmente por percusión, es decir, en las condiciones óptimas de la «Mecánica interna», ya bien conocidas y aplicadas en todo lo que sigue.

Cada impacto de bolas interesa, como término medio, una cantidad $q$ de materia tal que:

$$
q=\frac{W}{W_{m}} \text { o bajo otra forma : } q=K D^{3} .
$$

Sea un molino cerrado en el cual se estudia la variación de la superficie específica en función del tiempo. Hemos demostrado en un trabajo anterior ${ }^{*}$ ) que la curva obtenida puede representarse por la siguiente relación:

sabiendo que:

$$
\mathrm{S}_{p}=(1-A)^{a t}\left(\mathrm{~S}_{p i}+C\right)-C
$$

$\log =\log$ neperiano.

$$
a=\frac{7,5 K N \delta}{\alpha \pi \rho} \quad y \quad C=\frac{B W}{q \log (1-A)}
$$

Teniendo en cuenta la hipótesis efectuada sobre $q$, resulta:

$$
K=\frac{0,39_{\rho} g \varnothing_{\gamma}}{{ }^{\prime} W_{m}} \quad \text { de donde } \quad a=\frac{0,93 N g \varnothing_{\gamma} \delta}{\alpha W_{m}} \text { y } C=\frac{B W_{m}}{\log (1-A)}
$$

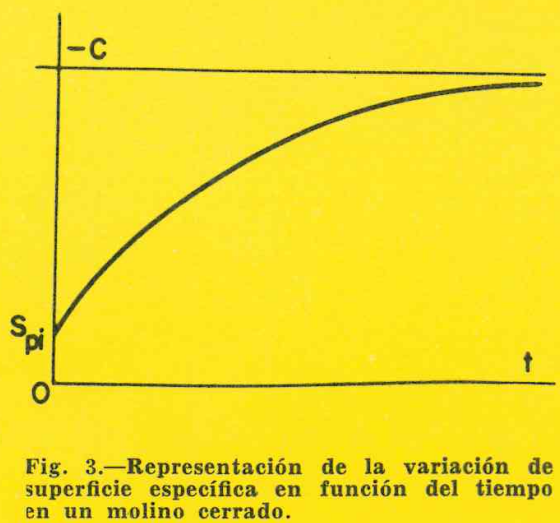

superficie específica en función del tiempo en un molino cerrado.

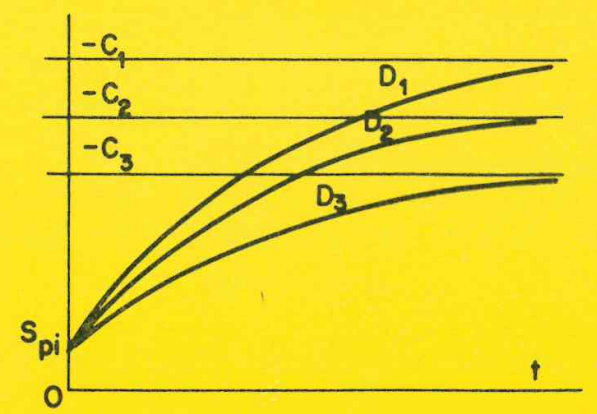

Fig. 4.-Influencia del diámetro de las bolas sobre la forma de las curvas.

(*) M. Papadakis: «Contribution à l'étude des broyeurs à boulets industriels». Revue des Matériaux de Construction, noviembre 1960, núm. 542. 
Sea ahora un molino de bolas, en el que se efectúan varias experiencias semejantes a la precedente, pero cambiando en cada caso el diámetro de las bolas. La experiencia demuestra que un material homogéneo suministra curvas tales como las de la figura 4, suponiendo $D_{1}<D_{2}<D_{3}$.

La única variable es $D$, y se encuentran claramente tres curvas diferentes si se emplean tres valores distintos de $D$. Ahora bien, $D$ no aparece directamente en la fórmula $[1]$. $D$ interviene indirectamente y sólo por los términos $\delta$ y $A$.

Establezcamos $p=\frac{0,93 N g \varnothing}{\alpha W_{m}}$, de donde se deduce que:

$$
a=p \gamma \delta
$$

La relación [1] puede escribirse:

$$
\begin{gathered}
\frac{\mathrm{S}_{p}+C}{\mathrm{~S}_{p i}+C}=(1-A)^{a t} \\
\circ \quad \log \left[\frac{\mathrm{S}_{p}+C}{\mathrm{~S}_{p i}+C}\right]=a t \log (1-A)=\frac{p \gamma \delta t B W_{m}}{C}
\end{gathered}
$$

de donde:

$$
\mathrm{r}=\frac{C \log \left[\frac{\mathrm{S}_{p}+C}{\mathrm{~S}_{p i}+C}\right]}{p \delta i B W_{m}}
$$

A las curvas $D_{1}, D_{12}, D_{3}$ corresponden, respectivamente, los términos $\delta_{1}, \delta_{2}, \delta_{3}$ y $A_{1}, A_{2}, A_{3}$, así como $C_{1}, C_{2}, C_{3}$, permaneciendo constantes los otros términos (y, por lo tanto, $\gamma$ ).

Se puede siempre elegir $D_{1}$, de modo que $\delta_{1}$ sea igual a 1 (tomando, por ejemplo, $D_{1}<\varnothing / 30$ ). Entonces es posible calcular $\gamma$ a partir de la fórmula [2] para la curva $D_{1}$ (es decir, a partir de los pares de valores $\mathrm{S}_{p} / t$ señalados sobre esta curva), puesto que todos los términos son conocidos. No se olvidará, efectuando este cálculo, que $C$ es negativo.

El valor de $\gamma$ encontrado conviene igualmente a las curvas $D_{2}$ y $D_{3}$, lo que permite entonces calcular $\delta_{2}$ y $\delta_{3}$. En efecto, no es malo precisar la función $\delta=F(D / \varnothing)$, pues ésta nos puede servir en otras ocasiones. Recordemos que es independiente de la naturaleza del material.

El examen de las curvas experimentales $D_{1}, D_{2}$ y $D_{3}$, suministra directamente los valores de:

$$
\begin{aligned}
& \log \left(1-A_{1}\right)=\frac{B W_{m}}{C_{1}} \\
& \log \left(1-A_{2}\right)=\frac{B W_{m}}{C_{2}} \text { etc... }
\end{aligned}
$$

Se ha tomado como hipótesis que $A$ :

- depende de la naturaleza de los materiales;

- crece con la energía de la bola, es decir, con $D$ y $\varnothing$.

Por definición, A está comprendido entre cero y uno.

Parece difícil conocer la forma exacta de la función que liga $A$ con las dos variables principales enunciadas anteriormente.

Precisamente, se puede sugerir una forma probable de esta función tal como:

$$
A=1-\frac{1}{e^{b \mathrm{D}^{m}}} \text { ó } \log (1-A)=-b D^{m}
$$


que responde a las condiciones:

$$
\begin{array}{ll}
A=0 & \text { para } D=0 \\
A=1 & \text { para } D=\infty
\end{array}
$$

Entonces resulta:

$$
\begin{aligned}
& \log \left(1-A_{1}\right)=-b D_{1}{ }^{m} \\
& \log \left(1-A_{2}\right)=-b D_{2}{ }^{m}, \text { etc... }
\end{aligned}
$$

de donde:

$$
m=\frac{\log \frac{C_{2}}{C_{1}}}{\log \frac{D_{3}}{D_{2}}}=\frac{\log \frac{C_{3}}{C_{1}}}{\log \frac{D_{13}}{D_{3}}}=\frac{\log \frac{C_{3}}{C_{2}}}{\log \frac{D_{2}}{D_{3}}}
$$

$m$ no puede ser conocida más que a partir de un conjunto de curvas tomadas 2 a 2 ( $m$ es una magnitud sin dimensiones).

$b$ se calcula fácilmente una vez conocida $m$.

En este estado de la teoría, el funcionamiento del molino cerrado está más o menos bien conocido, al menos cuando se ha de tratar un material homogéneo. La forma de las curvas y la posición de las asíntotas pueden preverse según la dimensión de las bolas empleadas y la na:uraleza de los materiales tratados, tal como lo demuestra el estudio experimental que sigue:

\section{Estudio experimental}

Se ha ensayado en un molino cerrado de $\varnothing=64 \mathrm{~cm}$, con bolas de acero:

$$
D_{1}=2 \mathrm{~cm} \quad ; \quad D_{2}=4 \mathrm{~cm} \quad ; \quad D_{3}=6 \mathrm{~cm}
$$

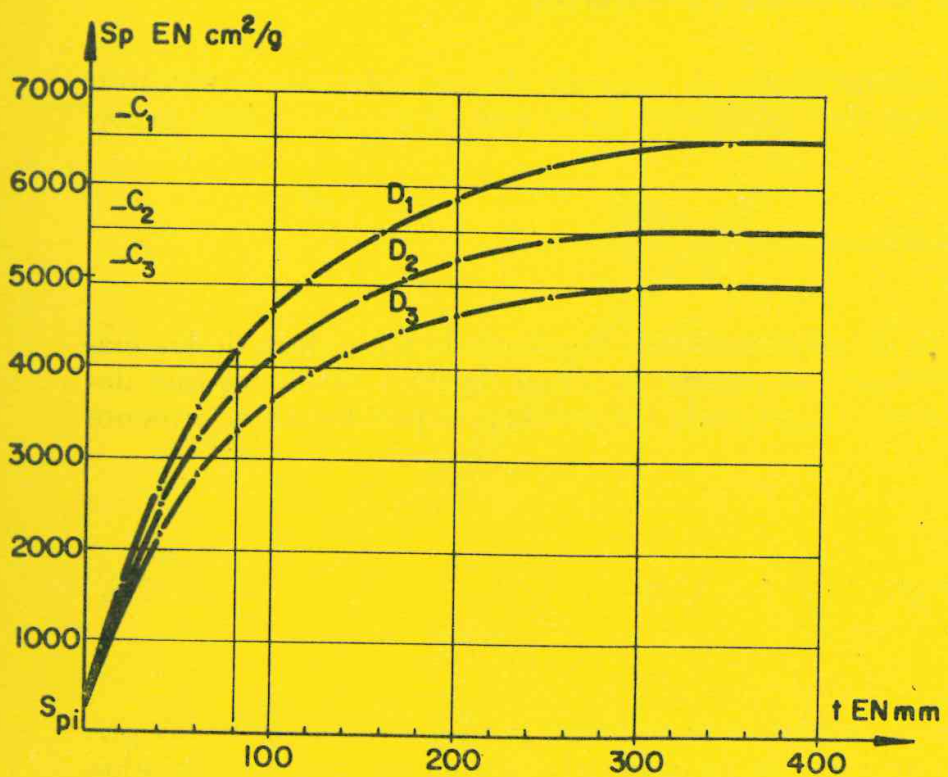

Fig. 5.-Ejemplo de molienda de clinker.

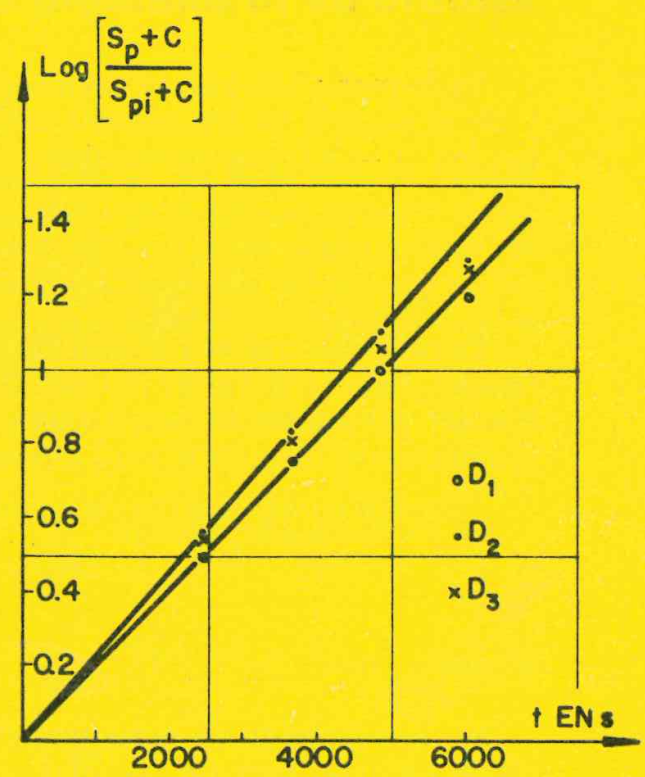

Fig. 6.-Justificación de la validez de la fó:mula [2]. 
un clínker para el cual:

$B=78 \times 10^{-7} \mathrm{~cm}^{2} /$ erg. Se ha tomado $\alpha=0,15$.

$W_{m}=10^{8} \mathrm{erg} / \mathrm{g}$.

Las curvas obtenidas relacionando la superficie específica y la duración de molienda, se representan en la figura 5 . Se observa que:

$$
-C_{1} \simeq 6.500 \mathrm{~cm}^{2} / \mathrm{g} ;-C_{2} \simeq 5.500 \mathrm{~cm}^{2} / \mathrm{g} ;-C_{3} \simeq 4.900 \mathrm{~cm}^{2} / \mathrm{g} .
$$

La aplicación de la fórmula [3] permite calcular $m$. Considerando las curvas 2 a 2 se encuentra que:

$$
\frac{\log \frac{C_{2}}{C_{1}}}{\log \frac{D_{1}}{D_{2}}}=0,24 \quad \frac{\log \frac{C_{3}}{C_{2}}}{\log \frac{D_{2}}{D_{3}}}=0,28 \quad \frac{\log \frac{C_{3}}{C_{1}}}{\log \frac{D_{1}}{D_{3}}}=0,26
$$

sea $m=0,26$. El valor de $b$ encontrado es $b=0,100$.

La fórmula [2] puede escribirse:

$$
\log \left[\frac{S_{p}+C}{S_{p i}+C}\right]=\frac{\gamma p \delta t B W_{m}}{C}=k t
$$

El término de la izquierda es proporcional al tiempo de molienda para un tamaño de bola dado Esto concuerda con lo representado en la figura 6, sobre la cual se ha llevado la relación entre $\log \left[\frac{\mathrm{S}_{p}+C}{\mathrm{~S}_{p i}+C}\right]$ y $t$.

Ensayos de la misma naturaleza efectuados sobre un molino de $\varnothing=36 \mathrm{~cm}$, han conducido a conclusiones semejantes, lo que permite creer en la validez de las fórmulas [1], [2] y [3].

\section{Estudio de la analogía entre molinos cerrados}

Ya hemos visto que el coeficiente de aglomeración, $A$, depende de los materiales a molturar y de la energía de los cuerpos molturadores. Este coeficiente interviene en el cálculo bajo la forma $\log (1-A)$, que se escribe:

$$
\log (1-A)=-b D^{m}=\frac{B W_{m}}{C}
$$

Es probable que $m$ y $b$ sean funciones de $\varnothing$ (permaneciendo constante la naturaleza de los materiales cuando $\varnothing$ varía). Parece difícil conocer, «a priori», la forma de estas funciones que deben ser precisadas de una manera experimental, aplicando los métodos expuestos anteriormente a molinos de diámetros muy diferentes.

Se escribirá simplemente:

$$
m=F(\varnothing) \quad ; \quad b=G(\varnothing)
$$

supuesto conocidas $F$ y $G$.

El conocimiento de estas funciones, cuya existencia se confirma por los resultados de los primeros ensayos, permite prever el comportamiento de un material dado en un molino «cerrado» de diámetro cualquiera. 
Se sabe que en tal molino, la relación entre superficie específica y tiempo no es jamás lineal, debiéndose la pérdida de energía, esencialmente, a la existencia del coeficiente de aglomeración $A$. El único medio de hacer la relación lineal consiste en reducir al máximo la influencia del coeficiente A. Para llegar a esto, es posible operar de dos maneras:

1) bien disminuir el diámetro del molino en función del tiempo (a condición de que $\delta$ quede próximo a 1);

2) bien disminuir el diámetro de las bolas en función del tiempo.

Habitualmente se eligió la segunda solución porque es más cómoda de aplicar.

Se demuestra entonces que si $D_{o}$ es el tamaño de las bolas correspondiente al tamaño die grano, $d_{o}$, del material al principio de la molienda, la repartición de tamaños viene dada por:

$$
D=\frac{D_{0}}{(a t+1)^{1 / m}}\left(\text { se recuerda que } \frac{W_{o}}{d_{o}^{2}}=C^{t e}\right)
$$

La relación entre $S_{p}$ y $t$ toma, entonces, la forma siguiente, deducida de consideraciones teóricas:

$$
S_{p}=\frac{B W_{m}}{a-\log \left(1-A_{o}\right)}(a t+1)+\left[S_{p i}-\frac{B W_{m}}{a-\log \left(1-A_{o}\right)}\right](a t+1) \frac{\log \left(1-\mathrm{A}_{o}\right)}{a}
$$

siendo $A_{0}$ el coeficiente de aglomeración correspondiente a $D_{o}$.

Cuando la $S_{p}$ elegida es muy superior a $\frac{B W_{m}}{a-\log \left(1-A_{o}\right)}$ puede emplearse la relación simplificada siguiente; en ella $a$ se desprecia frente a $\log \left(1-A_{o}\right)$, en tanto que la curva se asimila a su asíntota. Llamando $t_{s}$ al tiempo necesario tiene:

para obtener la superficie específica, $\mathrm{S}_{p}$, se ob-

$$
t_{s}=\frac{-\alpha\left[S_{p} \log \left(1-A_{o}\right)+B^{\prime} W_{m}\right]}{0,93 N g \varnothing \gamma B \delta} \text { con } \delta \simeq 1 \text { si } D_{o} / \varnothing \text { es pequeño. }
$$

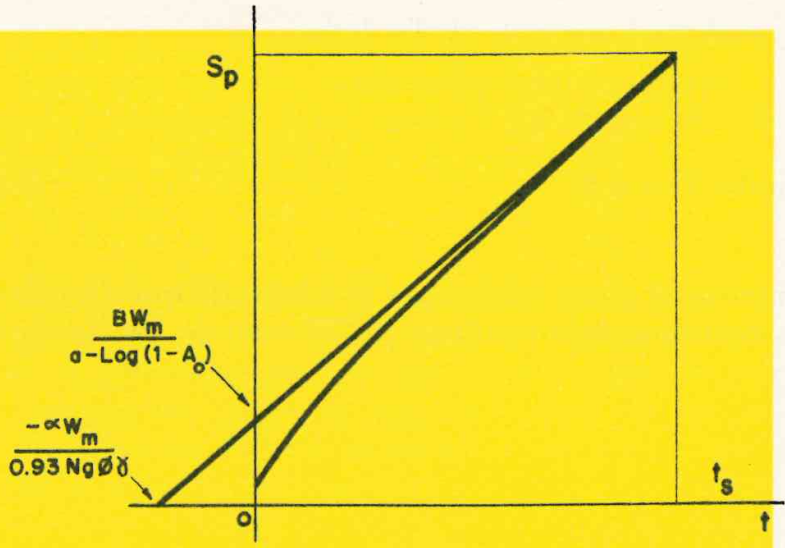

Fig. 7.-Establecimiento de la curva teórica $S_{p} / t$.
Nota.

La fórmula [4] es teórica. No puede verificarse rigurosamente, pues evidentemente es imposible hacer variar de una manera continua el tamaño de los cuerpos molturadores contenidos en el molino.

\section{Transposición al molino abierto}

En un molino «abierto», el material circula y la variable tiempo puede reemplazarse por la variable longitud. Todavía es aplicable el razonamiento hecho anteriormente. La superficie específica debe crecer linealmente con la longitud del molino. Este efecto puede obtenerse: 
1) disminuyendo el diámetro del molino de acuerdo con la longitud (de tal manera que $\delta$ permanezca igual a 1);

2) disminuyendo el tamaño de las bolas de acuerdo con la longitud.

Actualmente la solución más empleada es la segunda, pero la primera se ha utilizado también en alguna ocasión (molinos cónicos).

La circulación del material constituye un hecho nuevo. Ignoramos si este factor modifica el razonamiento propuesto para el molino cerrado $\mathrm{y}$, en caso afirmativo, cómo se introduce en los cálculos. Se puede enunciar la hipótesis de que el factor «circulación» modifica el término $\gamma$, coeficiente de transmisión de energía. Sería más difícil transferir energía de molienda a un material en circulación ( $y$, por lo tanto, constantemente renovado) que a un material no renovado. Sería preciso considerar un valor de $\gamma_{\mathrm{F}}$ para el molino cerrado y un valor de $\gamma_{o}$ para el molino abierto, tales que:

$$
\gamma_{0}<\gamma_{\mathrm{F}}
$$

Las bolas, por sí mismas, constituyen obstáculos que limitan la velocidad de movimiento del material molido.

Sólo experiencias repetidas, efectuadas sobre un molino abierto y con un material homogéneo, permitirán responder a esta cuestión.

Sea $t_{s}$ el tiempo necesario para obtener ia superficie $S_{p}$ (calculándose $a$ y $t_{s}$ a partir de $\gamma_{o}$, en el caso en que se adopte esta hipótesis). Entonces resulta:

$$
D=\frac{D_{o}}{\left(\frac{a t_{s} l}{L}+1\right)^{1 / m}}
$$

En estas condiciones el gasto teórico sería:

$$
Q=\frac{M}{t_{s}}
$$

$M=$ masa de material contenida en el molino;

$$
\begin{array}{ll}
\text { o bien: } & Q=\frac{0,47 \alpha T_{\rho} \phi^{2} L}{t_{s}} ; \quad T=\frac{\text { volumen aparente de las bolas }}{\text { volumen total del molino }} \\
\text { o bien: } & Q=\frac{0,19 \alpha_{\rho} \varnothing^{2} L}{t_{s}} \mathrm{~g} / \mathrm{s}, \text { tomando } T=0,40 .
\end{array}
$$

Sabemos que el gasto real será siempre inferior al gasto teórico por el hecho de que es prácticamente imposible asegurar una repartición rigurosamente continua del tamaño de los cuerpos molturadores.

Se puede establecer:

$$
r=\text { rendimiento del molino }=\frac{Q \text { real }}{Q \text { teórico }} ; r<1 .
$$

Sería necesario estudiar la importancia de la discontinuidad en el reparto del tamaño de los cuerpos molturadones sobre el valor de $r$. Ciertamente, este valor es muy importante. 


\section{Molinos industriales de circuito abierto}

El usuario del molino trata de sacar el mejor partido de su instalación, teniendo en cuenta el material que él trata. El constructor, generalmente, debe dimensionar un molino a partir de una muestra de material, con una producción y una finura impuestas.

Los problemas creados son, pues, un poco distintos y se les puede agrupar en dos tipos:

1) El molino existe ya; se trata de conocer la repartición óptima de bolas que permita obtener la máxima producción de material, fijada de antemano la finura de salida $S_{p}$.

Sea $\varnothing_{o}$ el diámetro al que corresponden $D_{0}$ y $b_{o}, m_{o}$ y, por lo tanto, $\log \left(1-A_{\sigma}\right)$. Se calcula $a$ y $t_{s}$ y se aplica la fórmula [5], obteniéndose:

$$
D=\frac{D_{0}}{\left(\frac{a t_{s} l}{L}+1\right)^{1 / m_{0}}}
$$

suponiendo que se pueden medir $B$ y $W_{m}$.

En el caso de que no se disponga de medios para medir $B$ y ${ }^{\prime} W_{m}$, se puede encontrar la repartición aproximada utilizando la fórmula [4] simplificada y la relación:

$$
B W_{m}=-C_{1} b_{1} D_{1}^{m_{1}}
$$

siendo $C_{1}$ el valor de la asíntota obtenida en un molino de laboratorio de diámetro $\varnothing_{1}$, con las bolas $D_{1}$. Entonces resulta:

$$
D=\frac{D_{o}}{\left[\left(\frac{S_{p} \log \left(1-A_{o}\right)-C_{1} b_{1} D_{1 .}^{m_{1}}}{C_{1} b_{1} D_{1}{ }^{m_{1}}}\right) \frac{l}{L}+1\right]^{1 / m_{o}}}
$$

2) El molino no existe todavía: no se puede proceder de otro modo más que dando las dimensiones $\varnothing$ y $L$ y calculando el gasto así obtenido. El cálculo se lleva como anteriormente para obtener $t_{s}$. A continuación se aplica la fórmula [6] afectada del coeficiente $r$, lo que da:

$$
Q_{\text {real }}=\frac{0,19 \alpha_{\rho} \phi^{2} L r}{t_{s}}
$$

Todo esto supone el conocimiento de las funciones $F$ y $G$ mencionadas en el apartado «Estudio de la analogía entre molinos cerrados». Si estas funciones dependen del material, es evidente que será preciso establecerlas para cada material mediante un estudio previo de laboratorio, efectuado sobre molinos de diámetros diferentes.

\section{Conclusiones}

Las investigaciones expuestas en este trabajo conducen a proponer métodos que permitan comprender mejor el funcionamiento de los molinos y de abordar los problemas de analogía. Este trabajo deberá continuarse haciendo variar sistemáticamente el factor «diámetro del molino» a fin de conocer con más precisión la forma de ciertas funciones. Sería también adecuado estudiar la influencia de la circulación del maierial sobre el funcionamiento de un molino experimental y deducir las leyes aplicables a los aparatos industriales.

El conjunto de los resultados obtenidos deberá permitir desarrollar un método más científico del cálculo de la producción de los molinos de bolas. 